Communications in Physics, Vol.28, No. 1 (2018), pp. 41-49

DOI:10.15625/0868-3166/28/1/10983

\title{
KINETIC MIXING IN MODELS WITH AN EXTRA ABELIAN GAUGE SYMMETRY
}

\author{
TRAN MINH HIEU ${ }^{\dagger}$ \\ Hanoi University of Science and Technology, 1 Dai Co Viet Road, Hanoi, Vietnam \\ ${ }^{\dagger} E$-mail: hieu.tranminh@hust.edu.vn \\ Received 17 December 2017 \\ Accepted for publication 17 January 2018 \\ Published 26 March 2018
}

\begin{abstract}
The Lagrangian of a theory possessing two Abelian gauge symmetries may contain a gauge kinetic mixing term without violating any known symmetries. In this paper, the formulation of theories extended by an additional Abelian gauge symmetry in the superfield formalism is presented. By rotating in the space of the gauge fields, the gauge kinetic mixing term can be eliminated, and the relevant charge and coupling are shifted in comparison with the original quantities. We demonstrate, for example, the effect of the gauge kinetic mixing term in the case of the $U(1)$ extension of the minimal supersymmetric standard model. Various physical observables are computed for a given benchmark point. We find that the gauge kinetic mixing is important for the model to predict the observables in a good agreement with experimental data.
\end{abstract}

Keywords: supersymmtry, Abelian gauge symmetry, kinetic mixing.

Classification numbers: 11.30.Rd; 12.60.Cn; 11.15.-q.

\section{INTRODUCTION}

The standard model (SM) is a successful theory in predicting experimental results. However, there are evidences that this model should be extended to explain experimental anomalies, and to address theoretical problems. To solve these issues, physics beyond the SM is necessary. Extensions of the SM usually imply the extension of the particle content, or that of the symmetry. If a grand unification theory (GUT) is assumed at high energies, there might be some remnant symmetries beside the SM gauge symmetry at lower energies. One possibility is that an additional $U^{\prime}(1)$ symmetry is left after the GUT gauge group is broken down [1,2].

(C) 2018 Vietnam Academy of Science and Technology 
In models with two Abelian gauge symmetries [3], in principle, the gauge kinetic mixing term of the form

$$
-\frac{k}{2} F_{\mu v}^{1} F^{2 \mu \nu}
$$

can exist in the Lagrangian since it is gauge invariant and does not violate any other symmetries. From the view point of a UV completion theory, even if the gauge kinetic mixing is set to zero at a high energy scale (for instant, the GUT scale), it can always be radiatively generated at lower energy scales via quantum corrections $[4,5]$. This term introduces one more degree of freedom to the theory compared to the case without the gauge kinetic mixing. The kinetic mixing term can be eliminated by an appropriate rotation in the space of Abelian gauge fields. This procedure results in the changes of the $U(1)^{\prime}$ charges and coupling. Therefore, it affects all the processes involving these quantities.

The influences of the gauge kinetic mixing have been discussed in literatures, see for instances [6] and [7]. Its role was shown to be quite important in certain cases. In this paper, we present a method to eliminating the gauge kinetic mixing term to get the canonical kinetic Lagrangian for the superfield formalism. This method is convenient when the gauge kinetic mixing parameter is considered as an input of the models. For demonstration, we investigate the effect of the gauge kinetic mixing in the $U(1)$ extension of the minimal supersymmetric standard model (UMSSM) which is inspired by the $E_{6}$ GUT. The physical observables including the SMlike Higgs boson mass, the muon anomalous magnetic dipole moment, the branching ratios of B-mesons rare decays, and the dark matter relic density are calculated. The results indicate that a non-zero kinetic mixing is necessary for the model to satisfy the experimental requirements.

The structure of the paper is as follows. In Sec. II, we briefly present the procedure to eliminate the gauge kinetic mixing term for non-supersymmetric (non-SUSY) theories. In Sec. III, the method is extended for the case of supersymmetric (SUSY) theories where superfields are invoked instead of normal fields. In Sec. IV, we analyze the effect of the gauge kinetic mixing in the UMSSM as a demonstration. Finally, Sec. V is devoted to conclusion.

\section{GAUGE KINETIC MIXING IN NON-SUSY THEORIES}

Assume that our theory has the gauge symmetry consisting of two Abelian gauge groups $U(1) \times U^{\prime}(1)$. The Lagrangian of the theory includes

$$
\mathscr{L}_{\text {gauge }}^{\text {kinetic }} \supset-\frac{1}{4} F_{\mu v}^{1} F^{1 \mu v}-\frac{1}{4} F_{\mu \nu}^{2} F^{2 \mu \nu}-\frac{k}{2} F_{\mu \nu}^{1} F^{2 \mu \nu},
$$

where $F_{\mu \nu}^{1}=\partial_{\mu} A_{v}^{1}-\partial_{v} A_{\mu}^{1}$ and $F_{\mu \nu}^{2}=\partial_{\mu} A_{v}^{2}-\partial_{v} A_{\mu}^{2}$ are the field strength tensors of the corresponding gauge fields $A_{\mu}^{1}$ and $A_{\mu}^{2}$. The last term is the so-called gauge kinetic mixing term that is Lorentz and gauge invariant, and $k$ is the mixing parameter.

To avoid this term, a rotation must be performed in the space of the two gauge fields:

$$
\left(\begin{array}{l}
A_{\mu}^{1} \\
A_{\mu}^{2}
\end{array}\right)=\left(\begin{array}{ll}
\frac{1}{\sqrt{2(1+k)}} & \frac{-1}{\sqrt{2(1-k)}} \\
\frac{1}{\sqrt{2(1+k)}} & \frac{1}{\sqrt{2(1-k)}}
\end{array}\right)\left(\begin{array}{l}
A_{\mu}^{Y} \\
A_{\mu}^{E}
\end{array}\right) .
$$

In order for the transformation to be real, the possible range for the kinetic mixing parameter is $-1<k<1$. This rotation induces a change in the gauge coupling matrix. This fact can be seen 
by considering the covariant derivative:

$$
\begin{aligned}
D_{\mu} & =\partial_{\mu}+i\left(\begin{array}{ll}
Y^{1} & Y^{2}
\end{array}\right)\left(\begin{array}{ll}
g_{11} & g_{12} \\
g_{21} & g_{22}
\end{array}\right)\left(\begin{array}{l}
A_{\mu}^{1} \\
A_{\mu}^{2}
\end{array}\right) \\
& =\partial_{\mu}+i\left(\begin{array}{ll}
Y^{Y} & Y^{E}
\end{array}\right)\left(\begin{array}{ll}
g_{Y Y} & g_{Y E} \\
g_{E Y} & g_{E E}
\end{array}\right)\left(\begin{array}{l}
A_{\mu}^{Y} \\
A_{\mu}^{E}
\end{array}\right),
\end{aligned}
$$

where the new $U(1)$ charges are exactly the same as the old ones, namely $Y^{1,2}=Y^{Y, E}$, and the new gauge coupling matrix is related to the original one by

$$
\left(\begin{array}{ll}
g_{Y Y} & g_{Y E} \\
g_{E Y} & g_{E E}
\end{array}\right)=\left(\begin{array}{ll}
g_{11} & g_{12} \\
g_{21} & g_{22}
\end{array}\right)\left(\begin{array}{ll}
\frac{1}{\sqrt{2(1+k)}} & \frac{-1}{\sqrt{2(1-k)}} \\
\frac{1}{\sqrt{2(1+k)}} & \frac{1}{\sqrt{2(1-k)}}
\end{array}\right) .
$$

After the rotation, we can verify that the $A_{\mu}^{Y}$ and $A_{\mu}^{E}$ have the canonical kinetic terms in the Lagrangian.

The matrix of the gauge couplings can be simplified further by the following orthogonal transformation without reintroducing the kinetic mixing:

$$
\left(\begin{array}{c}
B_{\mu} \\
E_{\mu}
\end{array}\right)=\frac{1}{\sqrt{g_{E E}^{2}+g_{E Y}^{2}}}\left(\begin{array}{cc}
g_{E E} & -g_{E Y} \\
g_{E Y} & g_{E E}
\end{array}\right)\left(\begin{array}{l}
A_{\mu}^{Y} \\
A_{\mu}^{E}
\end{array}\right) .
$$

The covariant derivative also changes accordingly

$$
\begin{aligned}
D_{\mu} & =\partial_{\mu}+i\left(\begin{array}{ll}
Y^{Y} & Y^{E}
\end{array}\right)\left(\begin{array}{ll}
g_{Y Y} & g_{Y E} \\
g_{E Y} & g_{E E}
\end{array}\right) \frac{1}{\sqrt{g_{E E}^{2}+g_{E Y}^{2}}}\left(\begin{array}{cc}
g_{E E} & g_{E Y} \\
-g_{E Y} & g_{E E}
\end{array}\right)\left(\begin{array}{l}
B_{\mu} \\
E_{\mu}
\end{array}\right) \\
& =\partial_{\mu}+i\left(\begin{array}{ll}
Y^{Y} & Y^{E}
\end{array}\right)\left(\begin{array}{cc}
g_{y} & g^{\prime} \\
0 & g_{E}
\end{array}\right)\left(\begin{array}{c}
B_{\mu} \\
E_{\mu}
\end{array}\right)
\end{aligned}
$$

where the new couplings are defined as

$$
\begin{aligned}
g_{y} & =\frac{g_{Y Y} g_{E E}-g_{Y E} g_{E Y}}{\sqrt{g_{E E}^{2}+g_{E Y}^{2}}}, \\
g_{E} & =\sqrt{g_{E E}^{2}+g_{E Y}^{2}}, \\
g^{\prime} & =\frac{g_{Y Y} g_{E Y}+g_{Y E} g_{E E}}{\sqrt{g_{E E}^{2}+g_{E Y}^{2}}} .
\end{aligned}
$$

The covariant derivative can be rewritten as

$$
\begin{aligned}
D_{\mu} & =\partial_{\mu}+i Y^{Y} g_{y} B_{\mu}+i\left(Y^{Y} g^{\prime}+Y^{E} g_{E}\right) E_{\mu} \\
& =\partial_{\mu}+i Y^{Y} g_{y} B_{\mu}+i e^{E} g_{E} E_{\mu},
\end{aligned}
$$

where we introduced the effective charge $e^{E}$ such that

$$
g_{E} e^{E}=Y^{Y} g^{\prime}+Y^{E} g_{E} .
$$

Here, $B_{\mu}$ plays the role of the ordinary gauge field associated with the hypercharge, while the extra gauge field $E_{\mu}$ will become massive after the $U^{\prime}(1)$ breaking. 


\section{GAUGE KINETIC MIXING IN SUSY THEORIES}

In SUSY theories, we work with superfields instead of normal fields. The gauge kinetic Lagrangian for the two Abelian vector superfields is written as

$$
\mathscr{L}_{\text {gauge }}^{\text {kinetic }} \supset-\int d^{2} \theta \frac{1}{4}\left(\begin{array}{ll}
W_{1}^{\alpha} & W_{2}^{\alpha}
\end{array}\right)\left(\begin{array}{cc}
1 & k \\
k & 1
\end{array}\right)\left(\begin{array}{l}
W_{1 \alpha} \\
W_{2 \alpha}
\end{array}\right)+\text { h.c. },
$$

where $k$ is the kinetic mixing parameter, and the SUSY field strengths are defined as

$$
W_{(1,2)}^{\alpha}=-\frac{1}{4} \bar{D} \bar{D} D^{\alpha} V_{(1,2)} \text {. }
$$

The kinetic mixing matrix can be diagonalized by a rotation among the original Abelian vector superfields:

$$
\left(\begin{array}{l}
V_{1} \\
V_{2}
\end{array}\right)=\left(\begin{array}{cc}
\frac{1}{\sqrt{2(1+k)}} & \frac{-1}{\sqrt{2(1-k)}} \\
\frac{1}{\sqrt{2(1+k)}} & \frac{1}{\sqrt{2(1-k)}}
\end{array}\right)\left(\begin{array}{l}
V^{Y} \\
V^{E}
\end{array}\right) .
$$

This rotation ensures that there is no explicit kinetic mixing in the Lagrangian written in the new basis $\left(V^{Y}, V^{E}\right)$. However, the effect of the kinetic mixing parameter will transfer to the interaction between the Abelian vector superfields and chiral superfields in the same way as in non-SUSY theories.

To see this, we consider the Lagrangian of the Abelian gauge interaction:

$$
\mathscr{L}_{\text {gauge }}^{\text {interaction }} \supset \int d^{4} \theta \Phi^{\dagger} e^{\mathbf{Q} \cdot \mathbf{g} \cdot \mathbf{v}} \Phi
$$

where

$$
\mathbf{Q} \cdot \mathbf{g} \cdot \mathbf{V}=\left(\begin{array}{ll}
Y^{Y} & Y^{E}
\end{array}\right)\left(\begin{array}{ll}
g_{Y Y} & g_{Y E} \\
g_{E Y} & g_{E E}
\end{array}\right)\left(\begin{array}{l}
V^{Y} \\
V^{E}
\end{array}\right)
$$

The gauge coupling matrix can be simplified by the following orthogonal rotation:

$$
\left(\begin{array}{l}
V^{Y} \\
V^{E}
\end{array}\right)=\frac{1}{\sqrt{g_{E E}^{2}+g_{E Y}^{2}}}\left(\begin{array}{cc}
g_{E E} & g_{E Y} \\
-g_{E Y} & g_{E E}
\end{array}\right)\left(\begin{array}{c}
V \\
V^{\prime}
\end{array}\right) .
$$

As a consequence, we have

$$
\mathbf{Q} \cdot \mathbf{g} \cdot \mathbf{V}=\left(\begin{array}{ll}
Y & Q^{\prime}
\end{array}\right)\left(\begin{array}{cc}
g_{y} & g^{\prime} \\
0 & g_{E}
\end{array}\right)\left(\begin{array}{c}
V \\
V^{\prime}
\end{array}\right)
$$

where we denote $Y=Y^{Y}$ and $Q^{\prime}=Y^{E}$.

For simplicity, we assume from now on that the original gauge coupling matrix is diagonal, $\operatorname{diag}\left(g_{1}, g_{1}^{\prime}\right)$. By the rotations (14) and (17), it becomes non-diagonal with the elements determined as follows

$$
\begin{aligned}
g_{y} & =g_{1}, \\
g^{\prime} & =\frac{-k g_{1}}{\sqrt{1-k^{2}}}, \\
g_{E} & =\frac{g_{1}^{\prime}}{\sqrt{1-k^{2}}} .
\end{aligned}
$$


We rewrite Eq. (18) in the form that will be convenient in the future:

$$
\mathbf{Q} \cdot \mathbf{g} \cdot \mathbf{V}=Y g_{y} V+Q^{p} g_{E} V^{\prime}
$$

where

$$
Q^{p}=Q^{\prime}-k \frac{g_{1}}{g_{1}^{\prime}} Y
$$

Here, we see that the gauge kinetic mixing affects the new gauge coupling $g_{E}$ and the new charge $Q^{\prime}$. Note that in the limit of vanishing kinetic mixing, $k \rightarrow 0$, the gauge coupling matrix becomes diagonal, and the redefined charge $Q^{p}$ equals the original one $Q^{\prime}$.

\section{EXAMPLE: THE UMSSM}

In this section, we consider the effect of the gauge kinetic mixing for an $U(1)$ extension of the minimal supersymmetric SM (MSSM). Beside the particles of the MSSM, the particle content of this model includes a vector superfield, $V_{2}=\left\{\tilde{B}^{\prime}, B_{\mu}^{\prime}\right\}$, corresponding to the additional $U^{\prime}(1)$ gauge symmetry, a singlet chiral superfield $S$ playing the role of breaking the extra $U^{\prime}(1)$ symmetry, and three generations of right-handed neutrino supermultiplets $N^{c}=\left\{\tilde{v}_{R}^{c}, v_{R}^{c}\right\}$.

The superpotential of the UMSSM includes terms from the MSSM superpotential and terms relevant to additional superfields:

$$
\left.\mathscr{W} \supset \mathscr{W}_{\mathrm{MSSM}}\right|_{\mu=0}+\lambda S H_{u} H_{d}+N^{c} \mathbf{Y}_{v} L H_{u} .
$$

The soft SUSY breaking Lagrangian reads

$$
\begin{aligned}
\mathscr{L}^{\text {soft }} \supset & \left.\mathscr{L}_{\mathrm{MSSM}}^{\mathrm{soft}}\right|_{B_{\mu}=0}-\left(\frac{1}{2} M_{1}^{\prime} \tilde{B}^{\prime} \tilde{B}^{\prime}+\tilde{v}_{R}^{c} \mathbf{A}_{v} \tilde{L} H_{u}+\text { h.c. }\right) \\
& -\tilde{v}_{R}^{c} \mathbf{M}_{\tilde{v}_{R}}^{2} \tilde{v}_{R}-m_{S}^{2}|S|^{2}-\left(\lambda A_{\lambda} S H_{u} H_{d}+\text { h.c. }\right) .
\end{aligned}
$$

The $U(1)^{\prime}$ charge of a chiral superfield is computed as

$$
Q^{\prime}=\cos \theta_{E_{6}} Q_{\chi}^{\prime}+\sin \theta_{E_{6}} Q_{\psi}^{\prime},
$$

where $\theta_{E_{6}} \in\left[-\frac{\pi}{2}, \frac{\pi}{2}\right]$ parameterizes the linear combination of two $E_{6}$ subgroups, $U(1)_{\chi}$ and $U(1)_{\psi}$ into the $U(1)^{\prime}$ symmetry. The charges $Q_{\chi}^{\prime}$ and $Q_{\psi}^{\prime}$ of the UMSSM chiral superfields are given in Table 1

Table 1. $U(1)^{\prime}$ charges of the UMSSM chiral superfields.

\begin{tabular}{|c|ccccccccc|}
\hline & $Q$ & $U^{c}$ & $D^{c}$ & $L$ & $N^{c}$ & $E^{c}$ & $H_{u}$ & $H_{d}$ & $S$ \\
\hline$\sqrt{40} Q_{\chi}^{\prime}$ & -1 & -1 & 3 & 3 & -5 & -1 & 2 & -2 & 0 \\
$\sqrt{24} Q_{\psi}^{\prime}$ & 1 & 1 & 1 & 1 & 1 & 1 & -2 & -2 & 4 \\
\hline
\end{tabular}


By the effect of the gauge kinetic mixing term, the redefined charges are shifted from the original ones as

$$
\begin{aligned}
Q_{Q}^{p} & =-k \frac{g_{1}}{g_{1}^{\prime}} \frac{1}{6}-\frac{1}{\sqrt{40}} \cos \theta_{E_{6}}+\frac{1}{\sqrt{24}} \sin \theta_{E_{6}}, \\
Q_{U^{c}}^{p} & =k \frac{g_{1}}{g_{1}^{\prime}} \frac{2}{3}-\frac{1}{\sqrt{40}} \cos \theta_{E_{6}}+\frac{1}{\sqrt{24}} \sin \theta_{E_{6}}, \\
Q_{D^{c}}^{p} & =-k \frac{g_{1}}{g_{1}^{\prime}} \frac{1}{3}+\frac{3}{\sqrt{40}} \cos \theta_{E_{6}}+\frac{1}{\sqrt{24}} \sin \theta_{E_{6}}, \\
Q_{L}^{p} & =k \frac{g_{1}}{g_{1}^{\prime}} \frac{1}{2}+\frac{3}{\sqrt{40}} \cos \theta_{E_{6}}+\frac{1}{\sqrt{24}} \sin \theta_{E_{6}}, \\
Q_{N^{c}}^{p} & =-\frac{5}{\sqrt{40}} \cos \theta_{E_{6}}+\frac{1}{\sqrt{24}} \sin \theta_{E_{6}}, \\
Q_{E^{c}}^{p} & =-k \frac{g_{1}}{g_{1}^{\prime}}-\frac{1}{\sqrt{40}} \cos \theta_{E_{6}}+\frac{1}{\sqrt{24}} \sin \theta_{E_{6}}, \\
Q_{H_{u}}^{p} & =-k \frac{g_{1}}{g_{1}^{\prime}} \frac{1}{2}+\frac{2}{\sqrt{40}} \cos \theta_{E_{6}}-\frac{2}{\sqrt{24}} \sin \theta_{E_{6}}, \\
Q_{H_{d}}^{p} & =k \frac{g_{1}}{g_{1}^{\prime}} \frac{1}{2}-\frac{2}{\sqrt{40}} \cos \theta_{E_{6}}-\frac{2}{\sqrt{24}} \sin \theta_{E_{6}}, \\
Q_{S}^{p} & =\frac{4}{\sqrt{24}} \sin \theta_{E_{6}} .
\end{aligned}
$$

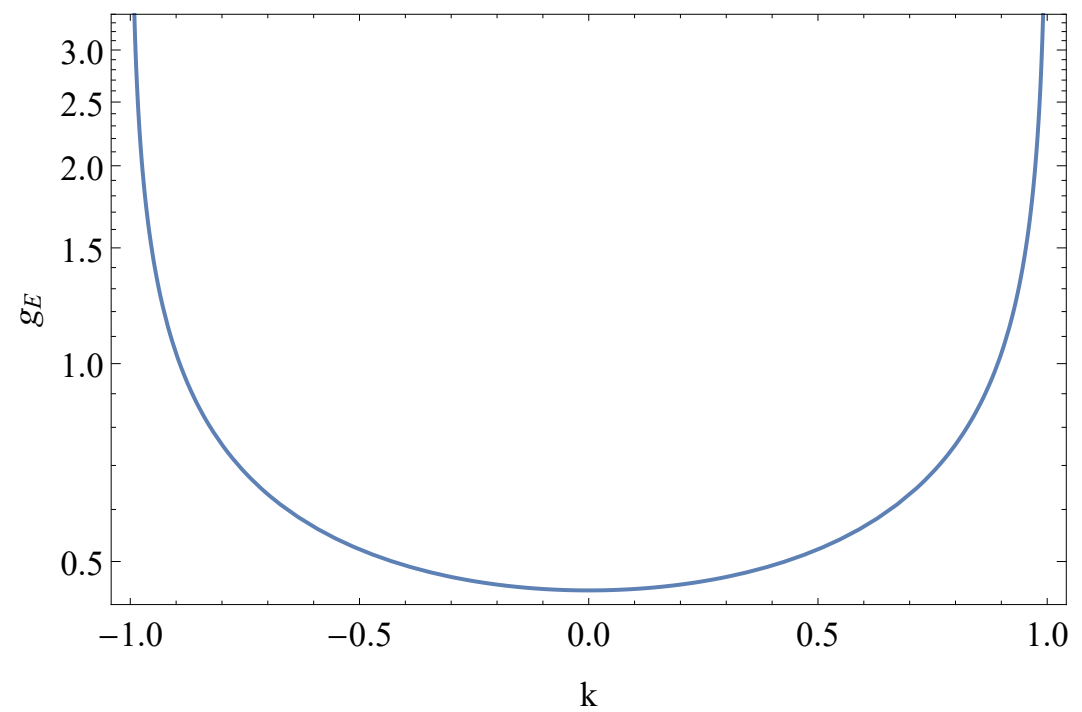

Fig. 1. The redefined gauge coupling $g_{E}$ as a function of the kinetic mixing parameter $k$. 
Since the UMSSM is inspired from the $E_{6}$ grand unified theory, we assume for simplicity that $g_{1}^{\prime}=\sqrt{\frac{5}{3}} g_{1}$. Figure 1 shows the dependence of the new gauge coupling $g_{E}$ on the kinetic mixing parameter $k$. We see that in the limit $k \rightarrow 0$ the redefined coupling $g_{E}$ becomes $g_{1}^{\prime}$. For large values of $|k|$, the coupling $g_{E}$ increases rapidly. In order for the theory to be in the perturbative regime, we must have

$$
\alpha_{E}=\frac{g_{E}^{2}}{4 \pi} \lesssim 1
$$

This constraint indicates that

$$
|k|<\sqrt{1-\frac{g_{1}^{\prime 2}}{4 \pi}} .
$$

In the benchmark analysis, we investigate the model with a given set of input parameters with non-zero gauge kinetic mixing:

$$
\begin{array}{rrrr}
M_{1}=87 \mathrm{GeV}, \quad M_{Z_{2}} & =5012 \mathrm{GeV}, \\
M_{2}=110 \mathrm{GeV}, \quad A_{0} & = & 3715 \mathrm{GeV}, \\
M_{3}=1800 \mathrm{GeV}, \quad \mu & =470 \mathrm{GeV}, \\
M_{1}^{\prime}= & 500 \mathrm{GeV}, \quad \alpha_{Z}= & 1.14 \times 10^{-4}, \\
m_{\tilde{1}}^{0}= & 650 \mathrm{GeV}, \quad \theta_{E_{6}}= & -1.419, \\
m_{\tilde{q}}^{0}=2200 \mathrm{GeV}, \quad k & = & 0.4037 .
\end{array}
$$

where $M_{1}, M_{2}, M_{3}, M_{1}^{\prime}$ are the gaugino masses; $m_{\tilde{l}}^{0}, m_{\tilde{q}}^{0}$ are the common inputs for slepton and squark masses; $M_{Z_{2}}, A_{0}, \mu$ are the $Z_{2}$ boson mass, the common input for trilinear couplings, the $\mu$-parameter, respectively; and $\alpha_{Z}$ is the Z-boson mixing angle. For this benchmark point, using the package MicrOMEGAs [8] we compute the SM-like Higgs boson mass, the muon anomalous magnetic dipole moment, the deflection of the electroweak $\rho$-parameter, and the branching ratios of the rare decays of B-mesons $\left(B_{s}^{0} \rightarrow \mu^{+} \mu^{-}, B^{ \pm} \rightarrow \tau^{ \pm} v_{\tau}, \bar{B}^{0} \rightarrow X_{s} \gamma\right)$. Assuming R-parity conservation, we find that the lightest neutralino is the dark matter candidate in this case, and compute its relic density. The values of these physical observables are as follows

$$
\begin{aligned}
m_{h} & =125.09 \mathrm{GeV} \\
\Delta a_{\mu} & =1.629 \times 10^{-9} \\
\Delta \rho & =9.9967 \times 10^{-5} \\
\operatorname{BR}\left(B_{s}^{0} \rightarrow \mu^{+} \mu^{-}\right) & =3.363 \times 10^{-9} \\
\operatorname{BR}\left(B^{ \pm} \rightarrow \tau^{ \pm} v_{\tau}\right) & =1.088 \times 10^{-4} \\
\operatorname{BR}\left(\bar{B}^{0} \rightarrow X_{s} \gamma\right) & =3.776 \times 10^{-4} \\
\Omega_{\tilde{\chi}_{1}^{0}} h^{2} & =0.1188 .
\end{aligned}
$$

The results are in good agreement with the present experimental constraints.

To study the effect of the gauge kinetic mixing, we perform a scan over $k$ while keeping all other parameters unchanged. In Figure 2, we show the muon anomalous magnetic dipole moment as a function of the kinetic mixing parameter. We can see the strong dependence on $k$ which helps to push up the value of $g_{\mu}-2$ to be within $2 \sigma$ of the measured value when $k \approx 0.4$. For this benchmark, the nonzero gauge kinetic mixing plays an important role to fit the experimental data. 


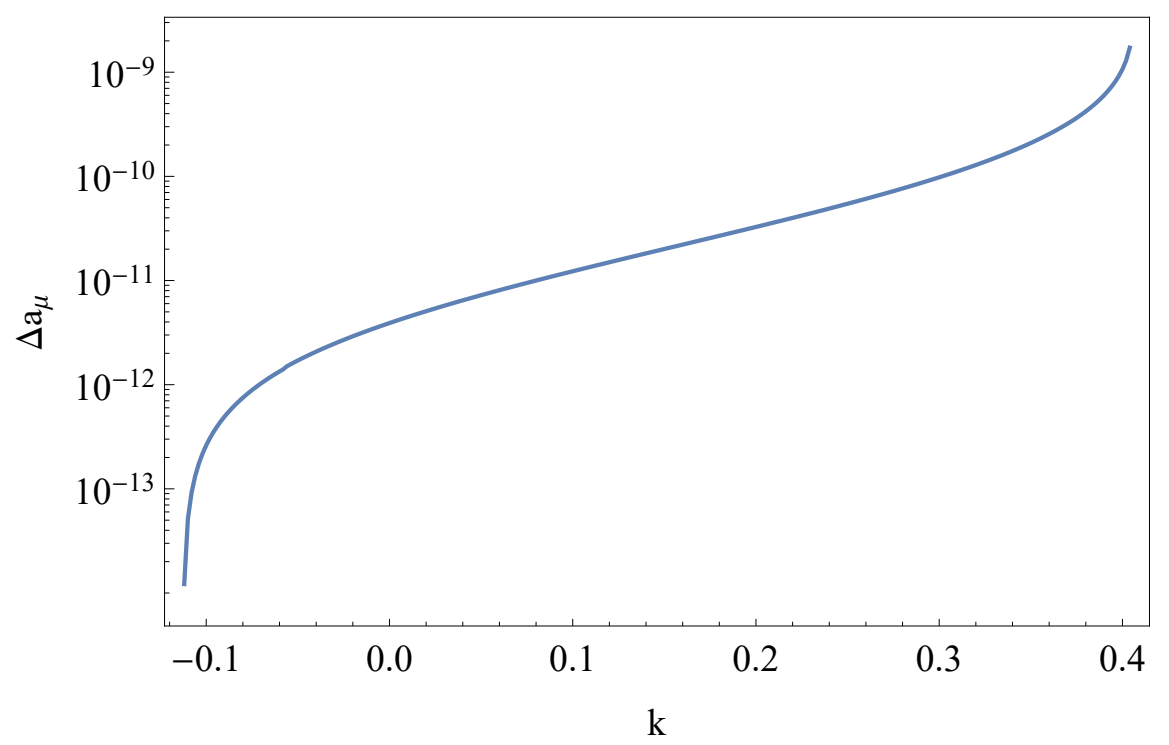

Fig. 2. The muon anomalous magnetic dipole moment $\Delta a_{\mu}$ as a function of the kinetic mixing parameter $k$.

\section{CONCLUSIONS}

In theories with two Abelian gauge symmetries, the gauge kinetic mixing term is allowed in the Lagrangian since it does not violate any known symmetries. Even if this term vanishes at a certain energy scale, it can always be induced radiatively at other scales by the renormalization group evolution. In this paper, we have presented a method to eliminate the gauge kinetic mixing term in the superfield formalism. As a consequence, this procedure leads to the changes in the new gauge coupling and the new $U^{\prime}(1)$ charges. The procedure has been applied to the UMSSM inspired from the $E_{6}$ GUT to study the effect of the gauge kinetic mixing term. We have analyzed the dependence of the new gauge coupling on the kinetic mixing parameter and derived its perturbative limit. We have computed physical observables for a given benchmark point and found that various phenomenological constraints are satisfied. With all other inputs fixed, a scan over $k$ showed the dependence of the muon anomalous magnetic dipole moment on the gauge kinetic mixing. In this case, a non-zero gauge kinetic mixing parameter is necessary to get the acceptable value for the muon $g-2$. Thus, the gauge kinetic mixing is an important factor to fit experimental results.

\section{REFERENCES}

[1] P. Langacker and J. Wang, Phys. Rev. D 58 (1998) 115010 [hep-ph/9804428].

[2] S. M. Barr and A. Khan, Phys. Rev. D 74 (2006) 085023 [hep-ph/0607054].

[3] B. Holdom, Phys. Lett. 166B, 196 (1986).

[4] F. del Aguila, G. D. Coughlan and M. Quiros, Nucl. Phys. B 307 (1988) 633 Erratum: [Nucl. Phys. B 312, 751 (1989)]. F. del Aguila, J. A. Gonzalez and M. Quiros, Nucl. Phys. B 307 (1988) 571 M. x. Luo and Y. Xiao, Phys. Lett. B 555 (2003) 279 [hep-ph/0212152];

[5] R. M. Fonseca, M. Malinsky, W. Porod and F. Staub, Nucl. Phys. B 854 (2012) 28 [arXiv:1107.2670 [hep-ph]]. 
[6] K. S. Babu, C. F. Kolda and J. March-Russell, Phys. Rev. D 57 (1998) 6788 [hep-ph/9710441].

[7] B. O'Leary, W. Porod and F. Staub, JHEP 1205 (2012) 042 [arXiv:1112.4600 [hep-ph]].

[8] G. Blanger, F. Boudjema, A. Pukhov and A. Semenov, Comput. Phys. Commun. 192 (2015) 322 [arXiv:1407.6129 [hep-ph]]; D. Barducci, G. Belanger, J. Bernon, F. Boudjema, J. Da Silva, S. Kraml, U. Laa and A. Pukhov, Comput. Phys. Commun. 222 (2018) 327 [arXiv:1606.03834 [hep-ph]]. 